ÉGYPTE monde arabe

\section{Égypte/Monde arabe}

33 | 1998

Travailleurs et industrie à l'heure des réformes

économiques

\title{
Tendances actuelles de l'industrie bancaire et nouvelle division internationale du travail
}

\section{Salwa El Antary}

Traducteur : Samia Rizk

\section{(2) OpenEdition}

\section{Journals}

Édition électronique

URL : https://journals.openedition.org/ema/1587

DOI : 10.4000/ema.1587

ISSN : 2090-7273

Éditeur

CEDEJ - Centre d'études et de documentation économiques juridiques et sociales

Édition imprimée

Date de publication : 30 juin 1998

Pagination : 225-253

ISSN : 1110-5097

\section{Référence électronique}

Salwa El Antary, «Tendances actuelles de l'industrie bancaire et nouvelle division internationale du travail », Égypte/Monde arabe [En ligne], 33 | 1998, mis en ligne le 08 juillet 2008, consulté le 07 juillet 2022. URL : http://journals.openedition.org/ema/1587 ; DOI : https://doi.org/10.4000/ema.1587

Ce document a été généré automatiquement le 7 juillet 2022.

Tous droits réservés 


\title{
Tendances actuelles de l'industrie bancaire et nouvelle division internationale du travail
}

\author{
Salwa El Antary \\ Traduction : Samia Rizk
}

\section{NOTE DE L'ÉDITEUR}

Les idées émises dans ce texte expriment le point de vue de l'auteur et pas forcément celui de son institution. Il s'agit d'une communication présentée dans le cadre du colloque «L'Egypte et le nouvel ordre économique mondial : la place de l'Egypte dans la division internationale du travail », organisé par le Centre d'études sur les pays en voie de développement et la Fondation Konrad Adenauer, les 28-29 avril 1998.

1 Malgré l'importance accordée aux recherches sur le mode de division internationale du travail et sur la nature de l'accumulation du capital à l'échelle mondiale dans la littérature sur l'économie mondiale (en tant que branche de l'économie politique), la majeure partie de ces écrits s'intéresse au premier plan à la division internationale du travail en termes de production de biens et, à un degré moindre, de services en général. Rares sont les études portant sur les services financiers qui s'intéressent, le cas échéant, à l'étude du degré de pertinence de la théorie des avantages comparatifs (cristallisée dans le modèle Heckscher-Holin) dans l'interprétation du commerce international des services. On sait sur quelles hypothèses théoriques, explicites ou implicites, cette littérature s'appuie: les relations économiques s'établiraient à l'échelle mondiale entre des pays de force équivalente, dans un monde dominé par la compétition. Ces hypothèses sont contraires à la réalité des relations internationales, contrôlées par les multinationales.

2 La question qui se pose à nous est de savoir si l'on peut réellement parler d'une division internationale du travail dans le secteur des services financiers? Et dans quelle mesure 
on peut s'appuyer sur les tendances de l'industrie bancaire dans ses pratiques effectives pour déterminer les indicateurs de cette division?

\section{État de l'industrie bancaire dans les pays développés}

3 L'étude de l'industrie bancaire au cours des deux dernières décennies montre que les banques commerciales des pays développés exercent leur activité dans le cadre d'un ensemble de facteurs qui, réunis, ont pour effet de réduire le taux de rentabilité. Les facteurs les plus importants sont :

$\left.41^{\circ}\right)$ la persistance des aspects de la crise structurelle de l'économie capitaliste, ce qui signifie l'incapacité de l'économie réelle ou effective à absorber tout le capital en numéraire disponible dans les banques. Cette crise peut s'appréhender à travers un ensemble d'indicateurs, notamment la baisse des taux de croissance et la hausse des taux de chômage (tableau 1). En effet, les politiques économiques des pays développés se sont attachées, depuis le milieu des années quatre-vingt, à lutter contre l'inflation, quitte à adopter des politiques de déflation aggravant le problème du chômage, notamment dans le cadre de la tendance croissante à l'automatisation ${ }^{1}$.

$\left.52^{\circ}\right)$ La tendance croissante des entreprises non bancaires à se lancer dans le domaine des affaires bancaires et à concurrencer les banques commerciales. La General Electric Capital, filiale de la firme américaine General Electric, en est un bon exemple (Canals, 1997, p. 6-7). Cette société finance le bail de machines et d'équipements aéronautiques ainsi que des projets dans l'industrie et le secteur du bâtiment-travaux publics (BTP); elle consent des crédits à la consommation par l'émission de cartes de crédit et la gestion des cartes de crédit émises par d'autres institutions. De plus, elle offre des services d'assurances, crée des fonds d'investissement et participe directement au capital des projets. Un autre exemple est celui de la multinationale ABB, fondée en 1987 par la fusion de la société suédoise ASEA et la société suisse Brown Bovery World Treasury. Pour servir ses objectifs, la société a créé un centre de financement offrant les services financiers qui lui sont nécessaires et visant plus particulièrement la mobilisation des ressources financières nécessaires à son expansion, au rachat d'autres entreprises ou à la création de nouvelles sociétés ('Abdallah, 1995).

$63^{\circ}$ ) L'orientation croissante du secteur des affaires vers l'émission d'actions et de titres sur les marchés de capitaux locaux et internationaux comme moyen de financement (Galpin, 1994)². Les résultats d'une étude comparative sur le mode de financement des sociétés industrielles dans un certain nombre de pays capitalistes développés indiquent que la proportion des emprunts bancaires par rapport au financement obtenu par ces sociétés a régressé durant la période 1983-1993 de $25 \%$ à $16 \%$ en France, de $25 \%$ à $21 \%$ en Hollande, de $46 \%$ à $35 \%$ au Portugal et de $33 \%$ à $27 \%$ au Royaume-Uni (Canals, 1997, p. 24).

$\left.74^{\circ}\right)$ Le déplacement des préférences des épargnants des dépôts bancaires à l'investissement en Bourse, directement ou à travers les fonds d'investissement, dans l'espoir d'obtenir un rendement plus élevé. Ces fonds ainsi que les autres formes d'investissement institutionnel constituent actuellement une source importante de financement du secteur des affaires par la souscription obligataire.

8 Le tableau 2 montre que les actifs financiers des sociétés d'assurances, des caisses de retraite et des sociétés d'investissement constituent à présent une part importante de 
l'ensemble des actifs des entreprises locales dans les pays développés (cette part varie entre $15 \%$ en Espagne et près de $55 \%$ aux États-Unis) et que cette proportion a connu une nette augmentation entre 1985 et 1995 au Canada, en France, en Allemagne, au Japon, en Norvège, en Espagne, au Royaume-Uni et aux États-Unis. Il se dégage également de ce même tableau que les dépôts du secteur des ménages auprès des canaux d'investissement institutionnel représentaient en 1995 pas moins de $24,4 \%$ en Espagne, $45,3 \%$ aux États-Unis et $52 \%$ au Royaume-Uni et que cette proportion a connu une forte augmentation durant la période 1985-1995 dans les principaux pays développés, notamment en Espagne, en France, en Allemagne, en Norvège et aux ÉtatsUnis.

9 Tous ces facteurs ont contribué à la baisse de la marge des taux d'intérêt et à la chute des taux de rentabilité des banques commerciales dans les grands pays développés notamment la France, l'Allemagne, le Japon et le Royaume-Uni (tableau 3).

\section{Tendances générales des activités bancaires}

Malgré la disparité et la diversité des stratégies adoptées par les banques commerciales en vue de maintenir leurs quotes-parts sur le marché du financement et de faire face à la chute de leur taux de rentabilité, ces stratégies présentent des points communs.

\section{L'augmentation des transactions sur le marché financier et le changement de nature des opérations d'intermédiation bancaire}

11 La stagnation économique et les faibles taux de croissance dans les pays développés entraînent pour l'investissement réel, et la sphère productive de manière générale, une incapacité à trouver les marchés de placement nécessaires pour absorber les capitaux en numéraire disponibles dans les banques commerciales; du coup, ces dernières ont dû chercher à réaliser des bénéfices en dehors du domaine de la production et se sont de plus en plus détournées de leur rôle initial qui consistait à fournir les capitaux nécessaires à l'économie réelle (financement et marketing de la production) pour s'orienter vers l'intermédiation bancaire, les services financiers et la gestion des risques, couvrant ainsi un certain nombre d'activités dont :

1. la gestion de l'émission des titres (actions et obligations);

2. la composition et la gestion de portefeuilles ;

3. la tenue des comptes de titres;

4. la souscription, le courtage et les opérations propres aux fonds d'investissement ;

5. les services relatifs aux opérations de fusion et d'achat des entreprises ;

6. le crédit-bail ;

7. les consultations financières ;

8. la spéculation dans les marchés des devises, des minéraux, des marchandises et de l'immobilier ;

9. la création de nouveaux instruments financiers qui acquièrent progressivement leur autonomie vis-à-vis du mouvement de l'économie réelle et qui font l'objet de transactions et de spéculations en Bourse tel que les instruments financiers dérivés ; 
10. la titrisation des créances existantes (notamment les dettes hypothéquées) et la mise en circulation de ces titres aussi bien à l'intérieur qu'à l'extérieur des marchés de capitaux, activité connue sous le nom de sécurisation.

12 Toutes les activités ci-dessus mentionnées figurent sur la liste des activités bancaires que les banques sont autorisées à exercer dans les différents pays de l'Union européenne, soit directement, soit par l'entremise de leurs filiales dans le pays hôte, dès lors qu'elles sont autorisées dans leur État d'origine, membre de l'Union (tableau 4).

De manière générale, dans les pays développés, les banques sont autorisées, à des degrés divers selon le pays, à exercer des activités relatives aux valeurs, aux biens immobiliers et à l'investissement dans des institutions non financières (tableau 5). Ces dernières peuvent d'ailleurs être propriétaires d'une banque. La seule exception réside en Irlande et au Japon, où les banques ne sont pas autorisées à entrer dans le domaine des assurances. Cette tentative des banques commerciales de diversifier leurs activités et de se lancer dans des activités de banques d'affaires a entraîné de nombreux amendements législatifs. Aux États-Unis par exemple (Canals, 1997, p. 135) ${ }^{3}$, le Glass Steagle Act de 1933, qui faisait une nette distinction entre les banques commerciales, d'une part, et les banques d'investissement et d'affaires, d'autre part, a été réinterprété : les premières ont été autorisées à se lancer progressivement dans les opérations de souscription et dans les transactions boursières à hauteur d'un pourcentage de leurs recettes ne dépassant pas $5 \%$ (en 1986), puis $10 \%$ (en décembre 1996), pour atteindre 25 \% des recettes en mars 1997.

De son côté, la Cour suprême américaine autorisait les banques (depuis 1996) à contracter des polices d'assurances (Canals, 1997, p.135). Quant aux restrictions législatives imposées à l'expansion des activités bancaires d'un État à l'autre, elles furent assouplies à compter de 1994 en vertu du Riegle Neal Act.

En outre, dans plusieurs pays européens, la législation fut amendée pour permettre aux banques de se lancer dans des activités de titrisation des créances. Ces amendements survinrent particulièrement au Royaume-Uni (1987), en France (1988), en Suède (1990) et en Espagne (1992)

\section{L'expansion des services électroniques}

16 Pour consolider leur position compétitive vis-à-vis des établissements non bancaires, les banques ont su tirer profit de l'énorme progrès réalisé dans le domaine de la technologie des communications et de l'informatique, aussi bien sur le plan quantitatif (nombre de services) que sur le plan qualitatif (moyens utilisés): équipements électroniques utilisés dans l'émission des chèques et autres modes de paiement, informatisation des prêts, de la gestion des biens et des transactions en Bourse. Notons par ailleurs la nette expansion des distributeurs automatiques de billets (DAB), des cartes de paiement électronique sur les points de vente et des services bancaires télécommuniqués par le biais d'« empreintes vocales » et du réseau internet ('Abd alRahîm, 1997)5.

\section{L'internationalisation des activités bancaires}

17 L'ouverture de leurs transactions à l'échelle mondiale constitue l'un des moyens mis en oeuvre par les banques pour affronter les facteurs ayant entraîné la baisse de leur taux 
de rentabilité à l'intérieur du pays. Cette tendance émerge fortement au cours des années soixante-dix, au moment où la liquidité, engendrée par les excédents du pétrole et par l'essor du marché de l'eurodollar, est un facteur important de l'intensification de l'activité bancaire dans le domaine du financement international et du recyclage des excédents pétroliers (El Antary, 1993, p. 195-206).

Toutefois, cette internationalisation s'est largement accélérée au cours des années quatre-vingt-dix avec la libéralisation croissante des marchés financiers locaux et internationaux et l'immense développement des moyens de communication et des technologies informatiques. On assiste parallèlement à un net changement au niveau des mécanismes de financement international: des crédits consentis aux gouvernements et aux institutions et garantis par ces derniers (modalité courante dans les années soixante-dix et au début des années quatre-vingt) on passe à l'émission de titres (obligations et actions) négociables sur les marchés financiers internationaux, ainsi qu'à la transformation des créances existantes en actifs financiers, facilement cessibles en Bourse dès l'apparition du moindre risque. Cette astuce, produit de la crise de l'endettement extérieur des pays du Tiers-Monde dans les années quatre-vingt, menace la situation financière des banques internationales créancières.

Malgré la continuelle augmentation en valeur absolue des crédits bancaires internationaux au cours des années quatre-vingt-dix, leur part relative par rapport à l'ensemble des modes de financement privé a régressé : de $35 \%$ en 1990, elle est passée à $20 \%$ en 1993 alors que, durant la même période, l'émission d'obligations passait de $63 \%$ à $72 \%$ et celle des actions de $2 \%$ à $8 \%{ }^{6}$. D'autre part, la majeure partie des crédits bancaires internationaux est consentie aux pays développés - notamment les États-Unis d'Amérique - pour le financement d'opérations de fusion et d'acquisition d'entreprises et de projets gigantesques. Ainsi, la part des pays développés dans les crédits syndiqués internationaux octroyés s'est accrue de près de $75 \%$ en 1992 à $85 \%$ en $1996^{7}$.

Ces changements se reflètent dans la structure des financements privés consentis aux pays du Tiers-Monde : leur part de crédits bancaires régresse de19,8 \% en 1990 à $12 \%$ en 1994. En revanche, on assiste à l'augmentation de leur part dans l'émission d'obligations (de 2,8 \% à $11 \%$ ) et d'actions (de $15,5 \%$ à 18,8 \%) durant la même période 8 . L'évolution de la structure du financement international privé démontre l'importance croissante des fonds d'investissement à l'échelle mondiale. Ceux-ci se transforment en établissements financiers géants déployant une grande activité dans la composition et la gestion de portefeuilles distribués sur les différents marchés de capitaux selon la rentabilité prévue.

21 Dans un tel contexte, le nombre de fonds d'investissement, destinés à opérer dans les marchés financiers créés entre 1990 et 1994, a quadruplé, atteignant 908, tandis que l'actif décuplé de ces fonds atteignait 132 milliards \$\$. Aux États-Unis seulement, le volume de leurs investissements en actions sur les nouveaux marchés varie entre 50 et 70 milliards $\${ }^{10}$.

Ces changements survenus dans la forme et les mécanismes de l'internationalisation des activités bancaires vont de pair avec le rôle grandissant des agences internationales privées de notation (Rating Agencies) dans la classification des États, des entreprises et des banques ainsi que des titres de financement qu'ils émettent; les notes attribuées par ces agences traduisent le degré de solvabilité de chacun de ces États et de ces établissements et contribuent ainsi à orienter les banques internationales et les différents organismes d'investissement institutionnel vis-à-vis de chacun d'eux ${ }^{11}$. 


\section{La fusion entre les banques} banques de détail (Retail) et de s'assurer ainsi une large base de déposants et, par conséquent, une source stable de financement de leurs activités. Les banques internationales avaient échoué dans ce sens lors de leur expansion dans les années soixante-dix, en raison des restrictions imposées par les législations locales de la plupart des pays hôtes en matière d'implantation des banques étrangères, et de la sévère compétition avec les banques locales, suffisamment fortes pour offrir des services bancaires de même niveau. Concernant les pays du Tiers-Monde, les rapports du Fonds monétaire international (FMI ${ }^{13}$ indiquent que les banques américaines et européennes ont acheté, durant la deuxième moitié des années quatre-vingt-dix, des participations de contrôle dans certaines banques d'Amérique latine et d'Europe de 
l'Est soit dans le cadre des privatisations, soit dans celui de la restructuration à la suite des crises financières qui y ont sévi. C'est ce qui se produisit en Argentine, au Brésil, au Mexique ainsi qu'en Hongrie et en Pologne.

Autant le mouvement de fusions et d'acquisitions conduit au développement des monopoles bancaires au niveau national, autant il réduit progressivement le nombre des principales banques internationales et les limites à quelques banques géantes qui, selon le FMI, ne sont pas plus que dix, parmi lesquelles six banques américaines ${ }^{14}$.

\section{Nouvelles tendances des activités des banques centrales}

31 La grande expansion des activités bancaires dans les pays capitalistes développés nécessitait de nombreux amendements législatifs autorisant les banques commerciales à étendre leurs activités à différents domaines (assurances, crédit immobilier, transactions en Bourse, participation directe au capital des projets), et éliminant de ce fait les différences traditionnelles entre banques commerciales, d'une part, et banques d'investissement et d'affaires, d'autre part. Par ailleurs, l'activité croissante de ces banques à l'échelle internationale nécessitait une réglementation garantissant :

1. la suppression de l'antagonisme entre la liberté de mouvement des banques internationales et les législations locales dans chaque État. C'est ce qu'assure le General Agreement on Tarifs and Trade (GATT) lors de l'Uruguay Round, puis la signature, le 13 décembre 1997, de l'accord multipartite relatif à la libéralisation des services financiers dans le cadre de l'Organisation mondiale du commerce ;

2. le contrôle des activités bancaires et des performances assurant une situation financière saine et prévenant la faillite. Ceci s'est révélé particulièrement important à la suite de la crise d'endettement des pays du Tiers-Monde envers les banques internationales durant la première moitié des années quatre-vingt, l'écroulement de la Banque de crédit et du commerce au début des années quatre-vingt-dix, puis de la Banque Barings en 1995. Cela montre les risques que comporte l'internationalisation des affaires bancaires et les mécanismes qui y conduisent.

Le comité de Bâle relevant de la Banque des règlements internationaux ${ }^{15} \mathrm{~s}^{\prime}$ est chargé de mettre en place le cadre organisationnel requis : un ensemble de normes et de règles unifiées régissant les affaires bancaires. Le FMI et la Banque mondiale sont chargés de contrôler le respect de ces normes et de ces règles par les différents États en les intégrant aux programmes de stabilisation et d'ajustement structurel contractés avec les pays qui le souhaitent.

\section{Normes internationales régissant les affaires bancaires}

\section{Normes d'adéquation du capital}

La Banque des règlements internationaux a posé les normes unifiées de l'adéquation du capital. Connues sous le nom de "Résolutions du comité de Bâle ", dont la déclaration date de juillet 1988, ces normes établissent un lien entre le capital de la banque, d'une part, et les actifs pondérés selon le degré de risques, d'autre part: le rapport entre le capital et ces actifs ne doit pas être inférieur à $8 \%$. En outre, tous les pays sont classés selon le degré de risques qu'ils présentent. Conformément à ce classement, les pays du 
Tiers-Monde, y compris les pays arabes - à l'exception de l'Arabie Saoudite - sont considérés comme des pays à hauts risques ${ }^{16}$. Les États membres du comité de Bâle se sont engagés à unifier les moyens de contrôle exercés par leur banque centrale sur les banques oeuvrant sur leur territoire et à relever progressivement l'adéquation du capital de celles-ci, pour atteindre $8 \%$ de l'ensemble des actifs à risque avant la fin de 1992.

Toutefois, l'application et le respect de ces normes ne se limitent pas aux États membres signataires des résolutions de Bâle; adoptées à l'échelle mondiale et en vigueur dans les différents systèmes bancaires, elles servent de base à la comparaison entre les banques et à l'évaluation de leur situation financière ${ }^{17}$.

\section{Règles unifiées du contrôle bancaire}

Lors des réunions du FMI et de la Banque mondiale en septembre 1997, le comité de Bâle sur le contrôle bancaire (The Basle Committee on Banking Supervision) a publié "Les principes fondamentaux d'un contrôle bancaire efficace " ${ }^{18}$, établis en collaboration avec les organismes de supervision et de contrôle du groupe des dix grands pays développés (G-10) et les marchés financiers émergents (notamment dans le Sud-Est asiatique) ainsi que le FMI et la Banque mondiale. Cette tâche répond aux recommandations du Sommet des sept plus grands pays développés (G-7) tenu en juin 1996 et concernant la nécessité d'unifier les normes et les règles de contrôle relatives à la gestion des marchés financiers au niveau mondial.

《Les principes fondamentaux de supervision bancaire efficace $~^{19}$ comporte vingt-cinq articles couvrant : les conditions que requiert sa mise en œuvre, à savoir la clarté et le respect des règles comptables universelles; les règles relatives aux autorisations et au contrôle de la structure du capital de la banque mère et des entreprises qui en relèvent ; la détermination des différents axes de supervision qui suppose des données, des systèmes informatiques et des politiques organisationnelles; la précision des règles de supervision de terrain et des modalités de vérification des données présentées par les banques concernant leur situation financière ; enfin, l'autorité dont bénéficient les contrôleurs et le moyen d'étendre la supervision aux activités des succursales et des filiales à l'étranger.

Le comité de Bâle a déclaré qu'il se chargeait de vérifier dans quelle mesure ces principes étaient appliqués par les différents États, et plus particulièrement par les marchés financiers naissants. De son côté, le FMI a déclaré que la sauvegarde du système bancaire devait figurer en tête des objectifs des autorités bancaires du monde entier ${ }^{20}$. À son tour, la Banque mondiale a insisté sur l'importance qu'elle accordait au soutien des instances de supervision dans les pays émergents aussi bien sur le plan législatif que sur le plan de la formation et du développement des capacités de supervision $^{21}$. En plus des normes internationales unifiées visant la régulation de l'activité bancaire, nous examinerons ci-dessus les principales tendances récentes concernant le rôle des banques centrales.

\section{La séparation de certaines fonctions}

Dans la pratique, les pays développés ont tendance à séparer l'autorité liée à l'élaboration de la politique monétaire et sa mise à exécution, de celle chargée de 
superviser le fonctionnement du système bancaire. En fait, ces deux types de fonctions ne se trouvent réunies sous la tutelle de la banque centrale que dans la moitié des pays développés seulement. Quoi qu'il en soit, dans l'ensemble de ces derniers, les fonctions relatives à la politique monétaire sont du ressort de la banque centrale, alors que celles liées à la supervision du système bancaire sont soit du ressort de la banque centrale, comme en Australie ou en Nouvelle Zélande, soit confiées à une autre instance, le plus souvent le ministère des Finances ou un organisme technique spécialisé comme c'est le cas au Japon, au Canada, en Autriche, en Suisse, en Belgique, au Danemark, en Norvège, en Allemagne et au Royaume-Uni (tableau 7). Aux États-Unis, le Federal Reserve Board, le ministère des Finances et la Federal Deposit Insurance Corporation se partagent la tâche.

Par ailleurs, parallèlement à cette tendance, en apparaît une autre: un même organisme est chargé de la supervision de tous les établissements financiers y compris les banques, les compagnies d'assurances et le marché des capitaux. En déclarant, en mai 1997, que la responsabilité de la supervision du système bancaire ne relevait plus de la Banque d'Angleterre mais du Securities and Investment Board (SIB) et maintenant, de la Financial Services Authority (FSA), le Royaume-Uni consacrait cette orientation $^{22}$. Cette même voie est suivie par des pays comme la Norvège où l'ensemble des activités du secteur financier, y compris les opérations bancaires, les assurances et les valeurs, est confié à une commission relevant du ministère des Finances : Banking, Insurance and Securities Commission ${ }^{23}$.

\section{La séparation entre les activités des banques centrales et la gestion de la dette publique}

Le FMI incite à séparer la gestion de la dette publique des activités de la banque centrale et à confier cette responsabilité à un organisme indépendant aux objectifs précis, s'appuyant sur des indices de performance mesurables et qui soit dirigé par des spécialistes. Les principales responsabilités de cet organisme sont de gérer au quotidien les risques encourus par le portefeuille de la dette publique, de fournir à l'État les liquidités nécessaires à un faible coût et à des conditions convenables, et de faciliter le recours de l'État aux marchés financiers internationaux en vue d'obtenir des financements en devises étrangères ${ }^{24}$. Selon le FMI, le fait de confier la gestion de la dette publique à un organisme indépendant et compétent permet à l'État de gagner la confiance des créanciers et d'être mieux évalué quant aux risques du crédit par les institutions internationales, ce qui lui permet d'obtenir des emprunts extérieurs à coût réduit et dans de meilleures conditions. Il est à signaler que cette tendance s'est concrétisée en Autriche, en Irlande, au Portugal et en Suède ${ }^{25}$

\section{Gestion professionnelle des réserves monétaires internationales}

41 Les textes de la Banque mondiale relatifs aux marchés émergents insistent sur l'importance, pour les banques centrales de ces pays, de disposer d'une bonne gestion de leurs réserves en devises étrangères, afin qu'elles puissent investir ces devises de manière rentable tout en poursuivant des objectifs de liquidité et en faisant face aux risques de fluctuation des taux de change et des taux d'intérêt ${ }^{26}$. 

étrangères accumulées par le flux de capitaux durant les années quatre-vingt-dix. Sur l'ensemble des flux de la période 1990-1996, estimés à près de 1,2 trillion \$, environ 575 milliards $\$$ (soit $45 \%$ ), accumulés sous forme de réserves internationales auprès des banques centrales, ont essentiellement été placés dans des actifs liquides à faible rendement, notamment les Bons du Trésor émis par le gouvernement américain. Nous pouvons en conclure que $49 \%$ des capitaux affluant sur les marchés émergents à la recherche d'un rendement élevé ont été rapatriés vers les marchés des pays développés et placés dans les actifs financiers à faible rendement. Le FMI estime que la différence entre les deux rendements représente un coût annuel de près de 10 milliards $\$$ que subissent les citoyens des économies naissantes ${ }^{27}$. bancaires ont produit des changements profonds au niveau du rôle des banques centrales: forte tendance à la suppression de certaines de leurs fonctions traditionnelles comme la détermination des normes de contrôle de la performance et des activités bancaires, et passage de ces fonctions à des institutions internationales à caractère supranational ou encore à des organes «professionnels » spécialisés comme dans le cas de la gestion de la dette publique et des réserves internationales. Cette dernière tendance précisément appelle une lecture attentive des textes annexes à «l'Accord général sur le commerce des services» concernant la libéralisation des services financiers.

L'article 1, paragraphe b de cette annexe stipule que les services importés dans le cadre de l'exercice du pouvoir étatique sont :

1. les activités exercées par une banque centrale ou une autorité monétaire ou toute autre entité publique en vue d'appliquer des politiques monétaires ou des politiques relatives au taux de change.

2. Les activités faisant partie de tout système officiel d'assurances sociales ou du système des retraites.

3. Toute autre activité exercée par une entité publique pour le compte du gouvernement, garantie par lui, ou utilisant ses ressources financières.

4. Autrement dit, les diverses activités exercées par la banque centrale échappent, par principe, à l'accord sur la libéralisation des services financiers dès lors qu'elles entrent «dans le cadre de l'exercice du pouvoir étatique » et qu'elles sont «fournies à titre non commercial, sans compétition avec un ou plusieurs prestataires de services» (article 1, paragraphe c-3 de l'accord sur la libéralisation des services financiers).

Le transfert d'une partie des fonctions des banques centrales à des organismes professionnels spécialisés permet par conséquent de compter ces fonctions au nombre des services financiers sujets à libéralisation, et de donner la possibilité à des sociétés, à des institutions et à des maisons d'expertise étrangères d'offrir leurs prestations dans ces domaines. L'article 1, paragraphe $c$ de l'annexe précise en effet que: «Dans l'application du paragraphe subsidiaire b-3 de l'article premier du présent accord, le terme "services" sera entendu comme incluant toutes ces activités, si l'un des membres autorise les prestataires de services financiers à exercer une activité quelconque parmi celles mentionnées aux paragraphes b-2 et b-3, et à entrer ainsi en concurrence avec une entité publique ou avec un prestataire de services financiers. » 


\section{Le conflit entre les composantes du capital bancaire}

Avec le développement de l'internationalisation des activités bancaires et des structures organisationnelles, la concurrence entre les composantes du capital bancaire international pour le partage du marché atteint son paroxysme, qu'il s'agisse de la pénétration de marchés autres, ou de la concurrence sur les marchés des pays du TiersMonde (marchés émergents), ou encore des tentatives de chacune des parties pour pénétrer les « zones d'influence traditionnelles » de l'autre partie.

Jusqu'en 1997, le Japon était un important fournisseur de capitaux sur la scène internationale. Nous pensons cependant que l'accord de Bâle concernant les normes d'adéquation du capital, et la tendance à standardiser les règles comptables à l'échelle internationale, puis la crise financière récente survenue dans le Sud-Est asiatique jouent un rôle primordial en matière de concurrence entre les composantes du capital bancaire au détriment des banques japonaises.

Avec la reconnaissance des règles de Bâle relatives à l'adéquation du capital, les banques japonaises se sont en effet trouvées face à :

1. un indice de solvabilité faible ne dépassant pas $3 \%$ (contre un minimum requis de $8 \%$ );

2. une classification du capital et des réserves ne tenant pas compte des profits résultant d'une revalorisation des titres au portefeuille des banques japonaises (les plus-values non réalisées), celles-ci n'étant pas considérées, contrairement aux règles comptables en vigueur au Japon, comme des réserves occultes pouvant figurer dans le capital de ces banques (alGawwâdî, 1997, p. 108) ${ }^{28}$.

Bien que le comité de Bâle ait accordé aux banques japonaises, provisoirement et à titre exceptionnel, le droit d'ajouter à leurs réserves $45 \%$ des plus-values non réalisées pour leur permettre de régulariser leur situation, il n'en reste pas moins que pour atteindre les indices de solvabilité internationaux, ces banques japonaises se trouvaient devant un dilemme : l'augmentation du capital, ou bien la réduction des actifs à risques (prêts et investissements financiers) - ce qui revient à limiter leurs activités.

50 À la suite de la baisse de l'indice Nikkei et la chute des prix à la bourse de Tokyo en 1991 (Millman, 1995, p. 142-146), la situation du capital des banques japonaises est la suivante :

1. pertes en capital proportionnelles à la baisse de la valeur du portefeuille au prix du marché, ce qui signifie l'érosion du capital des banques ;

2. difficulté de recourir à la Bourse pour augmenter leur capital étant donné la chute des prix des actions.

51 Quant à la deuxième alternative qui consiste à réduire les actifs à risques, il était difficile pour les banques japonaises de réduire leur portefeuille ( $11 \%$ de la totalité de leurs actifs en 1990) par la vente, alors que les indices boursiers étaient en baisse. Il ne restait donc plus qu'à réduire leurs activités de crédits sur le plan international et à se replier sur le Sud-Est asiatique.

52 Avec l'explosion de la crise financière dans les pays du Sud-Est asiatique depuis le deuxième semestre de 1997 et la forte baisse de l'indice Nikkei durant la période aoûtnovembre de la même année, les banques japonaises devenaient encore moins compétitives aussi bien à cause de l'érosion continuelle de leur capital que parce qu'elles étaient le principal créancier des banques de la région. À la fin de 1996, la part du Japon dans le crédit extérieur des pays du Sud-Est asiatique s'élevait à 35,4\% contre 
$6,3 \%$ pour les banques américaines et $43,2 \%$ pour les banques des pays de l'Union européenne ${ }^{29}$. D'autre part, les négociations relatives à un accord multilatéral sur les services financiers ont révélé les pressions exercées par les États-Unis en vue de pénétrer les marchés du Japon et du Sud-Est asiatique, notamment la Corée du Sud, ainsi que les pays d'Amérique latine (Higâzî, 1995).

Au cours des négociations de juin 1995, les États-Unis ont menacé de se retirer sous prétexte que « les engagements consentis par plusieurs États ne permettaient pas une ouverture réelle des marchés ». Le Conseil du commerce des services (qui relève de l'Organisation mondiale du commerce) s'est vu obligé de prolonger d'un mois les négociations, période au bout de laquelle il est parvenu (le 26 juillet 1995) à un accord provisoire, sujet à révision à la fin de 1997, après que le Japon et la Corée du Sud se sont engagés, derrière trente autres États, à améliorer les conditions d'accès des étrangers à leurs marchés.

Malgré cela, les États-Unis ont à nouveau émis des réserves et déclaré qu'ils maintenaient le droit d'imposer à l'avenir des restrictions à la pénétration de leurs marchés par les étrangers. En vertu de cet accord :

1. l'Union européenne s'engage à ouvrir ses marchés et à en autoriser l'accès sans restrictions sur la base du traitement préférentiel et du principe de la nation la plus favorisée, et de ne plus appliquer la clause de réciprocité durant la période couverte par l'accord.

2. Le Japon s'engage à transformer les accords bilatéraux conclus avec les Etats-Unis en accords multilatéraux, et à garantir l'accès des étrangers aux activités de gestion des caisses de retraite publiques et privées.

3. La Corée du Sud s'engage à relever le degré de pénétration de ses marchés par les banques étrangères, les compagnies d'assurances et les sociétés de Bourse, et à relever la part de participations étrangères autorisées de $10 \%$ à $15 \%$.

4. Le Brésil s'engage à autoriser la participation des banques étrangères à la privatisation de ses banques nationales et à ouvrir le marché des réassurances à la compétition étrangère.

$\mathrm{Au}$ cours de ses négociations avec le FMI pour l'obtention de crédits, la Corée a fait plus de concessions lors de la formulation définitive de l'accord en décembre 1997 : une plus forte présence de capitaux étrangers dans le domaine des caisses d'investissement, de toutes les formes de crédit-bail, de services relatifs aux titres et de conseils relatifs aux investissements étrangers ainsi que des sociétés d'évaluation des risques de crédit et des sociétés prestataires d'informations financières ${ }^{30}$.

Nous pouvons donc dire que les tendances de la compétition entre les composantes du capital bancaire international indiquent que la compétition se jouera essentiellement entre le capital américain, d'une part, et le capital européen, d'autre part, tout en tenant compte du privilège comparé des banques américaines (et britanniques) dans le domaine des activités bancaires et d'investissement et dans celui des services financiers électroniques.

L'exposé détaillé des principales tendances des activités bancaires et de celles des banques centrales dans les pays développés montre également que dans l'un et l'autre cas, la concentration sur les services, qui requièrent des compétences humaines spécialisées avec une composante technologique importante, aussi bien en communication qu'en informatique, est de plus en plus forte. L'industrie bancaire 
évolue par conséquent dans le sens d'une domination des banques multinationales essentiellement dans les domaines suivants :

1. les opérations de financement international, aussi bien par un mécanisme de prêts syndiqués que par les créances titrisées, les banques du Tiers-Monde étant maintenues dans le rôle du petit partenaire ponctuel, comme cela s'est produit avec les banques arabes durant l'époque de la manne pétrolière de la deuxième moitié des années soixante-dix et du début des années quatre-vingt (El Antary, 1993, p. 257-263).

2. La gestion des réserves des banques centrales en devises étrangères. L'Égypte, par exemple, confiait la gestion d'une partie de ses réserves internationales à la Federal Reserve Bank de New York. En 1995, la Banque centrale d'Égypte a annoncé qu'elle recevait les offres des différentes banques internationales pour la gestion d'une partie de ses réserves en devises étrangères et le placement de ces fonds dans les Bons du trésor, les obligations et les titres émis par les gouvernements étrangers ou garantis par eux (Al-Rifâ'i, 1995).

3. L'évaluation des risques de crédit des États, des établissements et des valeurs en Bourse.

4. Les consultations financières et juridiques relatives à la gestion de la dette extérieure. Notons à ce propos que le gouvernement coréen a eu recours, durant la dernière crise financière, aux entreprises américaines Goldman Sax et Salomon-Smith Barney, en tant que conseillers financiers. De même, les banques coréennes ont fait appel à un avocat international spécialisé dans les négociations relatives aux créances douteuses, auquel le Mexique avait fait appel lors de ses négociations avec les banques créancières ${ }^{31}$. Il est à signaler également que l'Égypte avait eu recours à Goldman Sax comme conseiller financier au sujet de la solvabilité des États, évaluation dressée par l'entreprise Moody's, et à Merrill Lynch pour celle dressée par Standard \& Poors.

5. Les systèmes électroniques de règlements des paiements interbancaires (tel que le système SWIFT auquel adhèrent de nombreuses banques égyptiennes et qui sert à envoyer les avis de paiement et autres avis bancaires sous une forme standardisée et reconnue par toutes les banques adhérant à ce système).

6. Les logiciels propres aux activités bancaires en réseau, aux transactions en devises étrangères et à la gestion des biens.

7. Les opérations bancaires d'investissement, notamment la gestion des fonds d'investissement et de services financiers liés à la gestion des portefeuilles (Custodian Services) pour le compte des banques internationales.

C'est dans ce contexte que l'on peut interpréter les déclarations du président du conseil d'administration de la Goldman Sax aux médias égyptiens les 22 et 23 avril 1998, où ce dernier exprime l'intérêt que porte son institution à se lancer sur le marché égyptien spécialement dans les secteurs des assurances et des communications, ou encore les négociations entre le ministère de l'Économie et cinq dirigeants de la Deutsche Bank au sujet d'une formule permettant à celle-ci d'accéder au secteur égyptien des assurances.

Reste enfin à se demander dans quelle mesure tout ce que nous venons d'exposer reflète une tendance à instaurer une division internationale du travail dans le domaine bancaire, division qui se forme et se précise sous le leadership des banques aux activités transnationales et qui est fondée sur la domination d'un marché capitaliste unique. 


\section{BIBLIOGRAPHIE}

\section{En arabe}

'ABDALLAH Ismâ̂̂l Sabrî, 1995, « al-Kawkaba » (La globalisation), dans Actes de la $19^{e}$ conférence scientifique annuelle des économistes égyptiens, Le Caire, al-gam'iyya al-misriyya lil-iqtisâd al-siyâsî wa-l-ihsâ' wa-l-tashrî' (Association égyptienne d'économie politique, de statistiques et de législation), 21-22 décembre.

'ABD AL-RAHÎM Sâmih Muhammad, 1997, al-Tigâra al-iliktrûniyya fí khidmat al-tigâra wa-l-masârif al-'arabiyya (Le commerce électronique au service du commerce et des banques arabes), Beyrouth.

AL-GAWWÂDÎ Zaynab, 1997, Âthâr ittifâqiyat al-GATT 'ala al-gihâz al-masrafî al-misri (Effets du GATT sur le système bancaire égyptien), thèse de magistère, Le Caire, Académie al-Sadat pour les sciences de l'administration.

Al-ittifâqiya al-'âmma lil-tigâra fî-l-khadamât (Accord général sur le commerce des services), 1994, silsilat awrâq lil-munâqasha, al-waraqa al-râbi'a, mars.

AL-RIFÂ'I Fayqa, 1995, « 'Awlamat al-riqâba al-masrafiyya (Mondialisation du contrôle bancaire) », Majallat al-bunûk, Ittihâd bunûk misr (Union des banques d'Égypte), mai-juin.

Banque centrale d'Égypte, al-Tatawwurât al-itimâniyya wa-l-masrafiyya 'an al-fatra min awwal yûlyu 1990hatta nihâyat yûnyu 1992 (Évolution des crédits et des activités bancaires du début juillet 1990 à fin juin 1992).

DUWIDÂR Muhammad, 1997, al-iqtisâd al-dawli (L'économie internationale), . Alexandrie, Dâr algâmi'a al-jadîda lil-nashr.

HIGÂZî Hasan, 1995, Athâr tahrîr al-tigâra al-dawliyya fî-l-khadamât al-mâliyya fî itar ittifaqiyyat gawlat Uruguay 'ala al-bunûk al-misriyya (Effets de la libéralisation du commerce international dans les services financiers dans le cadre des conventions de l'Uruguay Round sur les banques égyptiennes), Institut national de la planification, décembre.

\section{En langues étrangères}

ACIB Charles Sterer,-1994, Electronic Banking Developments, The Challenge of Global Cash Management, Banking Institute, Central Bank of Egypt, December.

ARNDT H. W., 1990, "Comparative Advantage in Trade and Financial Services », Banca Nazionale Del Lavoro, Quartely Review, March.

Banking Technology, « The Authority on Financial System Worldwide », London, October 1997.

BIS, 1997, Core Principles for Effective Banking Supervision, Basle, September.

CANALS Jordi, 1997, Universal Banking. International Comparison and Theoretical Perspective, Oxford, Clarendon Press.

EL ANTARY Salwa, 1993, Le rôle de l'activité bancaire dans la nouvelle forme d'intégration des économies arabes au marché capitaliste mondiale 1970 -1985, thèse de doctorat, Paris, Université de Paris VIII. 
Euromoney Publications, 1998, Euromoney, London, March.

FARRANCE Christopher, 1994, Plastic Money. Developments in Credit Cards, Banking Institute, Central Bank of Egypt, November.

GALPIN Rodney D., 1994, Developments in International Banking, Banking Institute, Central Bank of Egypt, July.

Institute of International Finance (IIF) :

- 1997a, Roundtable on Supervision in Emerging Market Economies, The Institute of International Finance, Inc, Washington DC, September.

- 1997b, Spreads and Risk in Emerging Markets Lending, 11 F Research Papers, December.

International Monetary Fund (IMF) :

- 1995a, Private Market Financing for Developing Countries, Washington DC, March.

- 1995b, International Capital Markets, Washington DC, August.

- 1997a, International Capital Markets, Washington DC, November.

- 1997b, « Interim Assessment, Crisis in Asia », World Economic Outlook, Washington DC, December.

- 1998, Toward a Framework for Financial Stability, Washington DC, January.

MILLMAN Gregory J., 1995, Around the World on a Trillion Dollars a Day. Kent, Pantam Press.

REID David, 1994, Central Bank Supervision. Lessons of the Past Decade, Banking Institute, Central Bank of Egypt, August.

Swiss Bank Corporation, 1995, Economic and Financial Prospects, June-July.

The World Bank, 1993, Financial Policy for Adjustment and Growth, volume 1, Egypt, September.

\section{ANNEXES}


Tableau 1 : Indicateurs de la crise structurelle dans les pays développés (\%)

\begin{tabular}{|c|c|c|c|c|}
\hline \multirow[t]{2}{*}{ Années } & \multirow{2}{*}{$\begin{array}{c}\text { Taux de croissance } \\
\text { du produit intérieur } \\
\text { brut réel }\end{array}$} & \multicolumn{2}{|c|}{ Taux de chômage } & \multirow[t]{2}{*}{ Taux d'inflation } \\
\hline & & $\begin{array}{c}\text { Groupe des pays } \\
\text { développés }\end{array}$ & $\begin{array}{c}\text { Pays de l'Union } \\
\text { européenne }\end{array}$ & \\
\hline $1960-1970$ & 4,1 & - & - & - \\
\hline $1970-1980$ & 2,4 & - & - & 9,5 \\
\hline $1980-1990$ & 2,4 & - & - & 4,3 \\
\hline 1990 & 2,5 & 6,2 & 8,1 & 5 \\
\hline 1991 & 0,8 & 6,9 & 8,6 & 4,5 \\
\hline 1992 & 1,7 & 7,8 & 9,9 & 3,4 \\
\hline 1993 & 0,9 & 8,2 & 11,2 & $\begin{array}{r}2,9 \\
\end{array}$ \\
\hline 1994 & 2,9 & 8,1 & 11,6 & 2,3 \\
\hline 1995 & 2,2 & 7,7 & 11,2 & 2,4 \\
\hline 1996 & 2,5 & 7,7 & 11,4 & 2,3 \\
\hline 1997 & 2,8 & 7,5 & 11,1 & 2 \\
\hline
\end{tabular}

Sources : IMF, World Economic Outlook, Interim Assessment, Washington DC, December 1997, p. 55, 56, 57 ; Banque mondiale, Rapport sur le développement dans le monde, 1992, p. 259 ; Banque mondiale, Rapport sur le développement dans le monde, 1995, p. 215.

Tableau 2 : Évolution du rôle des organes d'investissement institutionnel dans l'accumulation de l'épargne (\%)

\begin{tabular}{|c|c|c|c|c|}
\hline \multirow[t]{2}{*}{ Pays } & \multicolumn{2}{|c|}{$\begin{array}{l}\text { Part des actils de l'investissement institutionnel } \\
\text { dans les actlis des élablissements financiers } \\
\text { locaux (1) }\end{array}$} & \multicolumn{2}{|c|}{$\begin{array}{c}\text { Part des dépóts du secteur des ménages } \\
\text { auprès des organes d'investissement } \\
\text { instifutionnel dans fes actifs financiers de } \\
\text { ce secteur (2) }\end{array}$} \\
\hline & 1985 & 1995 & 1985 & 1995 \\
\hline Canada & 26.4 & 35,9 & 24,5 & 31,4 \\
\hline France (3) & $\$ 1,4$ & 23,4 & 15.7 & 27,3 \\
\hline Allemagne & 12,8 & 19 & 19.6 & 28,9 \\
\hline Japon (4) & 10,2 & 22,6 & 32,2 & 34,8 \\
\hline Nonège (5) & 13 & 21,9 & - 25,1 & 37,5 \\
\hline Espagne & 3,2 & $\$ 5$ & 2,9 & 24,4 \\
\hline Poyaume-Uni (6) & 26.7 & 31,6 & 0 & 52 \\
\hline Élats-Unis & 43,8 & 54,6 & 33,4 & 45,3 \\
\hline
\end{tabular}

Source : IMF, Intemational Capital Markets : Developments, Prospects and Key Policy Issues, Washington DC. November 1997, p. 135 (1) Pat des actils financiers des compagnies d'assurances, des tonds de retralte el des sociétés dinvestissement dans l'ensemble des actils financiers des établissements financiers locaux.

(2) Créances du secteur des ménages auprès des organes d'investissement institulionnel par rapport à l'ensemble des aclifs financierss du secteur des ménages.

(3) Données comportant exclusivement les aclifs des compagnies d'assurances et des socétés d'investissement

(4) Données comparatives pour l'année 1990 el non pour l'année 1985.

(5) Données plus récentes pour l'année 1993 et non pour l'année 1995.

(6) Données comparatives pour l'année 1988 e1 non pour lannèe 1985. 
Tableau 3 : Évolution de la rentabilité des banques commerciales dans les grands pays développés (\%)

\begin{tabular}{|l|c|c|c|c|c|c|}
\hline Pays & \multicolumn{2}{|c|}{ Intérêts nets / total des actlis } & \multicolumn{2}{c|}{ Autres revenus / total des actifs } & \multicolumn{2}{c|}{$\begin{array}{c}\text { Revenu réeVfonds } \\
\text { propres (1) }\end{array}$} \\
\hline & $1985-1989$ & $1990-1994$ & $1985-1989$ & $1990-1994$ & $1985-1989$ & $1990-1994$ \\
\hline Canada & 2,9 & 3 & 1,1 & 1,6 & 7,9 & 12,1 \\
\hline France & 1,9 & 1,2 & 0,5 & 0,8 & 0 & 3,3 \\
\hline Allemagne & 2,3 & 2,2 & 1 & 0,9 & 6,5 & 2,7 \\
\hline ltalie & 0 & 3 & 0 & 0,9 & 0 & 1,2 \\
\hline Japon & 1,2 & 1 & 0,3 & 0,1 & 10,4 & 1,5 \\
\hline GB (2) & 3,2 & 2,7 & 1,8 & 1,9 & 6,1 & 4,9 \\
\hline Etats-Unis & 3,5 & 3,7 & 1,5 & 2 & 5 & 8,5 \\
\hline
\end{tabular}

Source : IMF, Intemational Capital Markets : Developments, Prospects and Key Policy issues, Washington DC, November 1997, p. 147.

(1) Rendement après impôt divisé par le capital et les résenves à la lin de l'année précédente et auquel on déduit lindice des prix à la consommation.

(2) Oonnées relatives à ta période 1988-1989.

Tableau 4 : Activités bancaires soumises à la reconnaissance réciproque au sein de l'Union européenne (1)

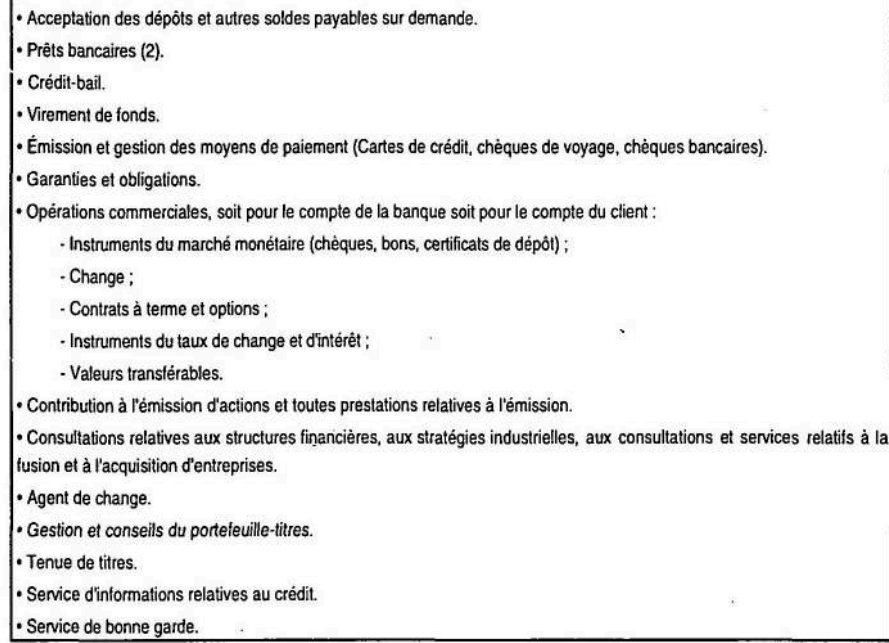

Source : IMF, International Capital Markets : Developments, Prospects and Key Policy Issues, Washington DC, November 1997, p. 185.

(1) The Second Banking Directive, en vigueur depuis le 1er janvier 1993, stipule que les banques de nimporte quel pays de I'Union europeeenne ont le droit d'exercer les activités ci-dessus mentionnées, directement ou par lintermédiaire de leurs succursales, dans les diftérents pays de lUnion dès lors que ces activités sont autorisées dans leur État d'origine. Ne figurent pas sur cette liste les activités relatives aux assurances et à limmobilier, celles-ci étant déterminées en fonction de la législation en vigueur dans le pays d'origine et dans le pays d'accueil.

(2) Y compris le crédit à la consommation, le prêt immobilier, le facturage (factoring) avec ou sans droit de recours et le financement des transactions commerciales ( $\mathrm{y}$ compris l'achat ou l'escompte des créances (forfaiting). 
Tableau 5 : Activités que peuvent exercer les banques commerciales dans les pays de l'Union européenne et dans le Groupe des 10

\begin{tabular}{|c|c|c|c|c|c|}
\hline Pays & Titres (1) & Assurancesa (2) & Immobilier (3) & $\begin{array}{c}\text { Investissement } \\
\text { dans des } \\
\text { ttablissemenls } \\
\text { non financiers }\end{array}$ & $\begin{array}{l}\text { Affiliation dे un } \\
\text { ottablistement } \\
\text { non financier }\end{array}$ \\
\hline \multicolumn{6}{|l|}{$\begin{array}{l}\text { Pays dont les } \\
\text { banques } \\
\text { exercent des } \\
\text { activités } \\
\text { extrèmement } \\
\text { étendues }\end{array}$} \\
\hline Australle & $\begin{array}{c}\text { Sans restrictions } \\
\text { (4) } \\
\end{array}$ & Autarisces (5) & Sans festiclions & Sans restrictions & Sans restrictions \\
\hline Suisse & Sans restrictions & Autorisés & Sans restrictions & Sans restrictions & Sans restrictions \\
\hline Royaume-Unt & Sans restrictions & Autorisées & Sans restrictions & Sans restictions & Sens restrictions \\
\hline France & Sans resurictions & Autonisées & Aulonistes & Sans restrictions & Sans restrictions \\
\hline Payseas & Sans restrictions & Autorisées & Auntorisées & Sans resurictions & Sans restrictions \\
\hline \multicolumn{6}{|l|}{$\begin{array}{l}\text { Pays dont les } \\
\text { banques } \\
\text { exercent des } \\
\text { activités } \\
\text { étendues } \\
\end{array}$} \\
\hline Danemark & Sans restrictions & Autorisees & Autorisées & Avec restrict. $\{6\}$ & Sans restrictions \\
\hline Finlande & Sans restictions & Avec restrictions & Autoristes & Sans restrictions & Sans restrictions \\
\hline Alemagne & Sans ressrictions & Avec restrictions & Autorisones & Sans sestrictions & Sans restrictions \\
\hline trelande & Sans restrictions & Interdites (7) & Sans restrictions & Sans restrictions & Sans restrictions \\
\hline Luxembourg & Sens restrictions: & Autoriśes & Sans ressuictions & Sens resvictions & Avec restictions \\
\hline Portugal & Sang restricions & Autorises & Avec restrictions & Autorisées & Autorisées \\
\hline Espagne & Sans restrictions & Aulorisees & Avec restrictions & Sans restictions & Avtorisees \\
\hline
\end{tabular}

\begin{tabular}{|c|c|c|c|c|c|}
\hline Pays & Titres (1) & Assurances (2) & Immobilier (3) & $\begin{array}{c}\text { Investissement } \\
\text { dans des } \\
\text { etablissements } \\
\text { non financiers }\end{array}$ & $\begin{array}{l}\text { Attliation à un } \\
\text { établissement } \\
\text { non financier }\end{array}$ \\
\hline \multicolumn{6}{|l|}{$\begin{array}{l}\text { Pays dont les } \\
\text { banques } \\
\text { exercent des } \\
\text { aetivités plutott } \\
\text { restreintes }\end{array}$} \\
\hline Halies & Sans restrictions & Autorisées & Avec restrictions & Avec restrictions & Avec restrictions \\
\hline Suède & Sans iestrictions & Autorisées & Avec restrictions & Avec restrictions & Avec restrictions \\
\hline Belgique & Autorisées & Autorisées & Avec restrictions & Avec restrictions & Sans restrictions \\
\hline Canada & Autorisées & Autorisées & Autorisées & Avec restlictions & Avec restrictions \\
\hline Groce & Autorisées & Avec restrictions & Avec restictions & Sans restrictions & Sans restrictions \\
\hline $\begin{array}{l}\text { Pays dont les } \\
\text { benquies } \\
\text { exercent des } \\
\text { activités } \\
\text { restreintes }\end{array}$ & *. & . & $\therefore$ & & \\
\hline Japon & Avec restrictions & Interdites & Avec restictions & Avec restrictions & Avec restrictions \\
\hline Elats-Unis & ecrest & Avec res: & Avec restrictions & Avec restrictions & Avec restrictions \\
\hline
\end{tabular}

Source : IMF, Intemational Capital Markets, November 1997.

(1) Titres : souscriptions, transactions, couriages, tonds dinvestissement

(2) Assurances : souscriptions à la vente des produits et des senvices relatilis aux assurances en tant que partie principale ov agent d'alfiaires.

(3) Immobilier : investissernent, développement et gestion.

(4) Sans restictions : les banques peuvent exercer directement les activités mentionnées.

(5) Autorisées : les banques peuvent exercer directement les activités mentionnées, entièrement ou en partie par lintermédiaire de tiliales.

(6) Avec restrictions : certaines des activités mentionnées peuvent être exercées, soil par la banque, soit par l'intermédiaire

des filiales.

(7) Interdites : les activités mentionnées ne petvent être exercées ni cirectement ni par le biais des flialeses. 
Tableau 6 : Mouvements de fusion et d'acquisition des banques dans les pays capitalistes développés

\begin{tabular}{|c|c|c|c|c|c|c|c|c|}
\hline \multirow[t]{2}{*}{ Pente } & \multicolumn{4}{|c|}{ Nonden dos: } & \multicolumn{4}{|c|}{ 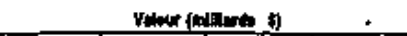 } \\
\hline & $\begin{array}{l}1000 . \\
1900\end{array}$ & 1081.1902 & 1003.10904 & $\begin{array}{c}1005-1805 \\
\text { (1) }\end{array}$ & 1900.1900 & $5001-1092$ & 1969.1506 & $\begin{array}{c}1985.1008 \\
\text { (1) }\end{array}$ \\
\hline \multicolumn{9}{|l|}{$\begin{array}{l}\text { Pays } 40 \\
\text { I'Union } \\
\text { nurop. }\end{array}$} \\
\hline Bangan & 16 & 22 & 18 & 12 & $=$ & 1 & 0,6 & 0.4 \\
\hline Futhands & 6 & 51 & 16 & 4 & 0.4 & 0,9 & 1 & 0.8 \\
\hline Frence & 32 & 1,33 & 11 & 43 & 2.7 & 2.4 & 0.5 & 3.2 \\
\hline Alamagne & w & 71 & 83 & 27 & 1.1 & 0,6 & 1,8 & 0.7 \\
\hline nate & 41 & 122 & 105 & 65 & 6.2 & 5,3 & 6,1 & 3. \\
\hline Ponstat & 12 & 20 & 13 & 7 & 10.9 & 0,1 & 0,1 & 0.8 \\
\hline Exposint & 30 & 76 & 14 & 26 & 1 & 4,3 & 4,5 & 2.1 \\
\hline Sulde. & 10. & 39 & 23 & 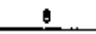 & 2 & 1.1 & 0,4 & 0.1 \\
\hline CP. & 46 & 71 & 40 & 20 & 5,4 & 1.5 & 3.3 & 21,7 \\
\hline \multicolumn{9}{|l|}{$\begin{array}{l}\text { Antres } \\
\text { Peye }\end{array}$} \\
\hline Anstales & 23 & 19 & 20 & $a$ & 2,3 & 0.5 & 4.5 & 2,5 \\
\hline Canada & t9 & 29 & 31 & 14 & 0.8 & 0,5 & $1, a$ & 0.1 \\
\hline Jypon. & 8 & 22 & 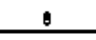 & 17 & 31.2 & - & 2.2 & 30.0 \\
\hline Norritge & 12 & 23 & 24 & 2 & 0,4 & 0.1 & 0.2 & 0.4 \\
\hline setsen & 31 & 47 & so & 14 & 0.5 & 0,4 & 3.0 & 0.7 \\
\hline Euts unis & 1501 & 1354 & $1 \mathrm{~km}$ & 1176 & 37,8 & Ss.s & 55.3 & 2,5 \\
\hline Tolla & 1655 & 2000 & 2032 & 1452 & 100.6 & $\$, 7$ & 03,2 & 153 \\
\hline
\end{tabular}

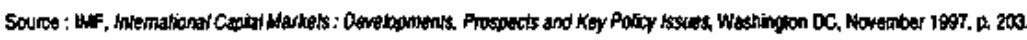
(1) Jusouten anil 1906

Tableau 7 : Autorités monétaires et autorités de contrôle bancaire dans les pays développés

\begin{tabular}{|c|c|c|}
\hline Peves & 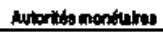 & 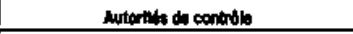 \\
\hline Australe & Bamque centidie & 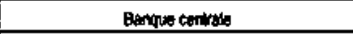 \\
\hline Auriche & Banque centriale & Minsistore des Finances \\
\hline Beniquive & Banque cemtirate & 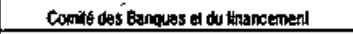 \\
\hline Canada & Banque centurie & Mhisthore des Finances \\
\hline Denemark & Banque centusle & Mristitere de rEcononile \\
\hline Finlande & Banque centrie & 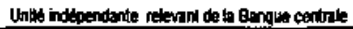 \\
\hline Frence & Banque centrale & Banque centula \\
\hline Allomagne & Ganque centrale & 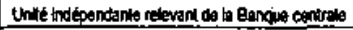 \\
\hline Grèce & Banque centrales & Banque centrols \\
\hline Heng Komg & Bancuperantrale & Bangue conirale \\
\hline Irelande & Banque centirle & 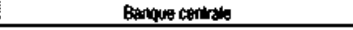 \\
\hline llalie & Banque centrale & Besque centirate \\
\hline Japon & Banque cenvile. & Banqua cerinate \\
\hline Luentourg & Banquecentiale & Banque centrie \\
\hline Pars-0as & Baneviscenturas & Banque cetrole. \\
\hline Nouvelezétande & Bampuo centrate & Bampincentions \\
\hline Nonege: & Aampe contries & Gampue centiole \\
\hline Pontuge: & Ganas centros & Ganerue centrab $\cdot=$ \\
\hline Espagra & Banque centrate & Banque centratie \\
\hline Subos & 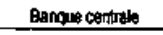 & 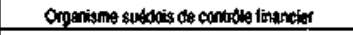 \\
\hline Suisse & Bancue centidio & Comites letitral beneatise \\
\hline foyaurpo-unt & Barque centrite & 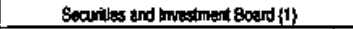 \\
\hline Élats-Unis & Banquat cenirile & 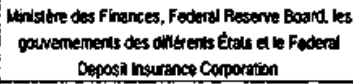 \\
\hline Venezueb & Banape contide & Heat Comato ducontrols bancaine \\
\hline
\end{tabular}

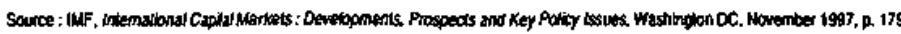

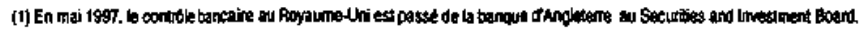




\section{NOTES}

1. Au sujet de la crise structurelle de l'économie capitaliste, crise marquée par les pressions inflationnistes structurelles cachées dans les replis de la stagnation à long terme, voir Duwîdâr (1997).

2. Swiss Bank Corporation (1995, p. 2-5).

3. IMF (1997a, p. 139) ; "Investment Banking -The New Retail Model», the Banker, London, Financial Times Business Ltd, April 1998, p. 17-20.

4. "Development of Securitization Around The World", The World of Banking, Lake Forest, USA, FIA Financial publishing Co., May-June 1993, p. 5-7 and 31.

5. «Beyond Virtual Banking ", Global Financial Review, USA, Unisys Corporation, Issue n 1,1998 ; Banking Technology, London,The Authority on Financial System Worldwide, October 1997 ; «Investment Banking... », op. cit., p. 81 - 86.

6. IMF (1995a, p. 15,24 et 72).

7. IMF (1997a, table 6, p. 11).

8. IMF (1995b, p. 25,42 et 46).

9. Institute of International Finance (IIF) (1997b, p. 1).

10. Ibid., p. 15.

11. IMF (1997a, p. 243). Il est intéressant de lire dans ce rapport que le nombre de pays que comporte l'évaluation Moody's a quintuplé entre la fin de 1989 et le début de 1996, et qu'il fournit la liste détaillée de ces évaluations en insistant sur l'intérêt qu'elles présentent pour faciliter l'accès de ces États aux marchés financiers internationaux. Le rapport s'appuie aussi sur les résultats obtenus par cette même institution dans son évaluation des systèmes bancaires dans les différents pays : voir p. 133-134.

12. E Banker, a supplement of The Banker, London, Financial Times Business Ltd, April 1998, p. 16.

13. IMF (1997a, p. 137-139).

14. Ces banques sont les suivantes: ABN Amro (Hollande), City Corp. (États-Unis), Barklays (Royaume-Uni), Deutche Bank (Allemagne), Goldman Sax (États-Unis), G.B. Morgan (États-Unis), Morgan Stanley (États-Unis), Merrill Lynch (États-Unis), S.B.C. Warburg, Banque fédérale suisse (Suisse). Source : FMI (1997a, p. 206).

15. Le comité de Bâle a été formé en 1975 par décision des gouverneurs des banques nationales des dix plus grands pays développés (G-10). Chargé de la supervision des activités bancaires, ce comité est constitué des représentants des instances de contrôle bancaire et des banques centrales de Belgique, du Canada, de France, d'Allemagne, d'Italie, du Japon, du Luxembourg, de Hollande, de Suède, de Suisse, du Royaume-Uni et des États-Unis. Il se réunit au siège de la Banque des règlements internationaux dans la ville de Bâle, en Suisse.

16. À ce sujet, voir : Ittihâd al-masârif al-'arabiyya, muqarrarât lajnat bâzil hawla kifâyatra's al-mâl (Union des banques arabes, résolutions du comité de Bâle sur l'adéquation du capital), Beyrouth, 1990 ; Al-bank al-ahHal-misrî, al-nashra al-iqtisâdiyya (Banque nationale d'Égypte, Bulletin économique), $\mathrm{n}^{\circ}$ 3,1988, p. 182-183.

17. Par décision du conseil d'administration de la Banque centrale égyptienne à la séance du $17^{\circ}$ janvier 1991, les banques égyptiennes sont obligées d'appliquer les normes d'adéquation du capital définies par le comité de Bâle. Le délai accordé aux banques pour régulariser leur situation et atteindre les $8 \%$ requis s'étendait jusqu'au $31^{\circ}$ décembre 1995 ; délai qui fut rapproché par la suite au 31 décembre 1993. Voir à ce sujet : Banque nationale d'Égypte, altatawwurât al-i'timâniyya wa-l-masrafiyya 'an al-fatra min awwal yûlyû 1990 natta nihâyat yûnyû 1992 (Évolutions des crédits et des activités bancaires pour la période du début juillet 1990 à la fin juin 1992), circulaire $\mathrm{n}^{\circ} 310 / 4$ en date du $14^{\circ}$ février 1991, p. 131-133.

18. IMF (1998, p. 52-70) ; IIF (1997a) ; AL-RIFÂ'Î (1997, p. 26-29).

19. BIS (1997). 
20. IIF (1997a, p. 51).

21. IIF (1997a, p. 55).

22. IMF (1997a, p. 179).

23. Ibid.

24. Ibid., p. 219-220.

25. IMF (1997a, p. 23-27).

26. The World Bank (1993, p. 80-82).

27. IMF (1997a, p. 222-227)

28. IMF (1997b, p. 26).

29. Ibid., Table 2, p. 7.

30. WTO, www.wto.org/wto/new/sumfin.htm, $17^{\circ}$ December 1997, p. 10-12.

31. Euromoney Publications (1998, p. 32-37).

INDEX

Mots-clés : banques, système bancaire

\section{AUTEURS}

\section{SALWA EL ANTARY}

Centre de recherches de la Banque nationale d'Égypte 